\title{
Enfermería de práctica avanzada: praxis, políticas y profesión*
}
Advanced practice nursing:
Praxis, policy and profession

\section{Enfermagem de prática avançada: prática, política e profissão}

Recibido: 19 de octubre de 2019. Aceptado: 08 de mayo de 2020. Publicado: 30 de junio de 2021.

DOI: https://doi.org/10.11144/Javeriana.rgps20.epap

\author{
Ricardo A. Ayala ${ }^{a}$ \\ Ghent University, Bélgica \\ ORCID: https://orcid.org/0000-0001-7840-1072 \\ Pierre Pariseau-Legault \\ Université du Québec, Canadá \\ ORCID: https://orcid.org/0000-0001-9833-0187
}

Para citar este artículo: Ayala RA, Pariseau-Legault P. Enfermería de práctica avanzada: praxis, políticas y profesión. Rev Gerenc Polit Salud. 2021;20. https://doi.org/10.11144/Javeriana.rgps20.epap

\footnotetext{
* Artículo de investigación.

a Autor de correspondencia. Correo electrónico: RicardoAlexis.AyalaValenzuela@UGent.be
} 


\section{Resumen}

En este artículo presentamos un análisis crítico de la transformación que ha tenido la profesión de enfermería luego de la institucionalización de la práctica avanzada (EPA). Hacemos un repaso del desarrollo histórico de la EPA principalmente en Estados Unidos y Canadá-, y discutimos las implicancias culturales y legales para la práctica de enfermería y para las enfermeras como sujetos sociales. En tanto esta reflexión permite planear una nueva agenda de investigación en el área de la salud, surge una preocupación sobre la contradicción interna en torno a la medicalización, como también sobre la necesidad de evaluar cuidadosamente la transferencia de esta política a otros sistemas de salud.

Palabras clave: Política de salud, salud pública, legislación, enfermería, Canadá.

\section{Abstract}

This article summarizes and critically discusses recent policy changes in the nursing profession, usually known as advanced practice nursing. We review its historical development - with an emphasis on the USA and Canada - the making and the implications of the policy for the healthcare system, and the cultural and legal repercussions for health, nursing practice, and for nurses as social agents. While these insights are useful for setting a new agenda for health research, it also raises issues about internal contradictions with regard to medicalization, as well as the need to carefully evaluate the process of policy transfer.

Keywords: Health policy, public health, legislation, nursing, Canada.

\section{Resumo}

Este artigo faz uma análise crítica da transformação pela qual passou a profissão de enfermagem após a institucionalização da enfermagem de prática avançada (EPA). É feita uma revisão do desenvolvimento histórico da EPA - principalmente nos Estados Unidos e Canadá-, e são discutidas as implicações culturais e legais para a prática de enfermagem e para as enfermeiras como sujeitos sociais. Embora esta reflexão permita o planejamento de uma nova agenda de pesquisa na área da saúde, ela também levanta a preocupação sobre a contradição interna em torno da medicalização e sobre a necessidade de avaliar cuidadosamente a transferência da política para outros sistemas de saúde.

Palavras-chave: Políticas de saúde, saúde pública, legislação, enfermagem, Canadá. 


\section{Introducción}

Pocos cambios recientes han despertado tanto optimismo entre los reformadores de la salud como la expectativa de formar un(a) profesional de práctica avanzada. Si bien ha recibido varias denominaciones tales como enfermera de práctica avanzada (advanced practice nurse/infirmière en pratique avancée), enfermera especialista (nurse practitioner/infirmière praticienne spécialisée) o super enfermera (super nurse/super infirmière), todos los nombres apuntan a una progresión o expansión del campo ocupacional, especialmente a partir de tareas y roles delegados, lo que generalmente se percibe como beneficioso para la salud y para la enfermería.

Como política sanitaria, la enfermería de práctica avanzada (EPA) fue ideada para favorecer el acceso y la sostenibilidad de un sistema que enfrenta cada vez más necesidades de salud y restricciones de personal (1-3). Sin embargo, hasta ahora los efectos de la política han sido evaluados parcialmente. Es relevante discutir sus aspectos centrales pues en la transferencia de una política subyace el supuesto de que su implementación acarreará los beneficios que ha tenido en el lugar de origen y que servirá para mejorar las políticas existentes (4).

En tanto este análisis permite planear una nueva agenda de investigación sobre las profesiones de salud, advierte también la necesidad de adaptar la política a las necesidades específicas del lugar donde se transfiera.

\section{Definición y contexto sociohistórico}

\section{Práctica avanzada: definición y contexto}

De acuerdo con la Asociación de Enfermeras de Canadá (5), EPA es un término genérico que incluye:

tanto la enfermera (registered nurse) como la enfermera especialista (nurse practitioner), cuya formación incluye tanto un programa de nivel superior, como una experticia y conocimientos clínicos en profundidad que permiten tomar decisiones complejas a fin de satisfacer las necesidades de individuos, familias, grupos, comunidades y poblaciones (5 p.13).

Como mínimo se requieren estudios oficiales de nivel superior además de una formación clínica en un campo de especialización (6). Por tanto, en Canadá, como en Estados Unidos, varias categorías de enfermería se agrupan bajo el término EPA, incluyendo la de nurse practitioner (enfermera especialista con atribuciones médicas) ${ }^{1}$ y la de clinical nurse specialist (enfermera clínica que estudia una especialidad de enfermería) ${ }^{2}$. Este estudio se centra en la categoría de nurse practitioner con especialización en atención primaria, ya que la amplia literatura sobre su ejercicio permite reflexionar mejor sobre sus implicancias. 
Para la fecha de este estudio, Canadá cuenta con unas 5.697 enfermeras de práctica avanzada (7), mientras que los Estados Unidos con unas 270.000 (8), se caracterizan por un rol ampliado y según las provincias y zonas en que ejercen y en función de su especialidad pueden realizar pruebas de diagnóstico, prescribir tratamientos médicos, remitir al usuario a otros profesionales y servicios de salud, y bajo determinadas condiciones definir ciertos problemas $(7,9)$. Aunque la mayoría de las enfermeras especializadas en Estados Unidos ejerce en el nivel primario de atención también suelen trabajar en hospitales y residencias para personas mayores, de manera que se pueden enfocar en especialidades tan diversas como salud mental, pediatría, geriatría o neonatología.

Este modelo de EPA es resultado de fuerzas sociales e históricas que han caracterizado la organización del sector salud en Norteamérica, incluyendo, como señalan MacDonald et al. (10):

las necesidades de salud de población, la oferta y demanda de profesionales de la salud, las políticas gubernamentales, [la evolución de] los currículos educativos, la colaboración entre enfermeras y miembros de otras profesiones, la evaluación [positiva] del papel de la enfermera en práctica avanzada y la economía de la salud (10 p.173).

Aspectos sociales en especial los movimientos por los derechos civiles erigieron temas como el género y la etnicidad en la lucha por reivindicar el trabajo del cuidado (10). En Norteamérica la conciencia sobre conflictos armados como la Segunda Guerra Mundial y la Guerra de Vietnam ha contribuido al reconocimiento del papel de las enfermeras en práctica avanzada (10,11). En Estados Unidos las enfermeras de anestesia y las enfermeras obstétricas estuvieron entre los primeros grupos de EPA (12), mientras que el modelo canadiense estaba destinado principalmente a los territorios rurales que no disponían de cuidados ni atención primaria $(10,12)$. En Estados Unidos el primer programa para nurse practitioners inició en 1965 (10), mientras que en Canadá los primeros programas se crean luego del informe Boudreau en 1972, el cual recomendaba el despliegue de nurse practitioners en todo el país a fin de abordar necesidades primarias de salud (11). Así, la integración relativamente reciente de la EPA refleja el deseo de reformar la atención primaria y luego la secundaria y terciaria, para responder a las dificultades de acceso a la salud de muchos ciudadanos (13).

No obstante, este proyecto también ha tenido dificultades. Su evolución ha gravitado principalmente hacia zonas geográficas no atendidas por médicos y especialidades con escasez significativa de médicos $(12,14)$. Por consiguiente, los obstáculos para la expansión de roles enfermeros se explican precisamente por tratar de suplir esa escasez, además de otros aspectos sociopolíticos y económicos durante las reformas (14,10). Así también, las presiones políticas en la década de 1970 desencadenaron una disminución del desarrollo de la práctica avanzada en Canadá encontrando opositores fuera y dentro de la profesión. Por ejemplo, algunas enfermeras académicas consideraron que la EPA sólo constituía una estrategia de sustitución del personal médico; consideremos que la comunidad de enfermería tenía muchas dificultades para definir el 
papel de práctica avanzada (10) y por supuesto, desde la profesión médica también se originaron presiones políticas que afectaron el avance del proceso (10).

En tanto esta problemática ilustra el contexto de la práctica avanzada es necesario indagar en su historia para comprender mejor sus contornos y matices, y con ello ponderar la viabilidad de transferir o replicar este modelo a otros sistemas de salud.

\section{La práctica avanzada en la historia}

El desarrollo de la EPA ha sido un proceso heterogéneo. La institucionalización de la enfermería durante el siglo pasado fue una historia de delegación desde la profesión médica, que se interesaba en nuevos métodos y tecnologías. Gran parte de la práctica denominada 'propia' tiene su origen en ese proceso (15), lo que hace que la práctica actual también esté en constante transformación tanto para auxiliares y técnicos como para las enfermeras profesionales que avanzan en un doble frente: en el plano gerencial y en terapias invasivas. Procesos de fisión, fusión, mutación, adopción y asimilación siguen caracterizando el modelaje continuo de las fronteras profesionales $(15,16)$ en los cuales es muy reveladora su relación con la medicina como profesión dominante.

Además, la industrialización temprana de Canadá y Estados Unidos (17) marcó la adopción precoz de roles médicos de parte de las enfermeras, en particular debido a la migración interna de la población hacia los centros urbanos. La concentración en centros industriales conlleva una centralización de servicios dejando las zonas agrarias o remotas atendidas por personal de postas y dispensarios. A ello se suma la reactivación del mundo rural en la década de 1930 pero sin servicios para las zonas alejadas. En tanto el personal debía lidiar con problemas médicos y quirúrgicos urgentes, esto permite suponer que la delegación antecede al concepto de práctica avanzada.

Los registros más tempranos en Norteamérica provienen de la segunda mitad del siglo XIX, cuando las enfermeras realizaban case management (18), administraban anestesia inhalatoria y prescribían medicinas en situaciones urgentes (19). No obstante, con el cambio de siglo y al expandirse fuera de las áreas desposeídas, la situación se complicaba para la profesión médica en tanto los servicios de la enfermera parecían emular a los del médico, al tiempo que la economía de Estados Unidos se agravaba y hacía incierto el escenario salarial del médico (20). Si bien la movilización de los médicos durante esa economía inestable fue fructífera, las intervenciones médicas ejecutadas por enfermeras continuaron desarrollándose informalmente. En especial en países menos industrializados se realizaban también tratamientos no médicos que a veces requerían fármacos (21). Era común también que durante las urgencias las enfermeras oficiaran de segundo cirujano o, como era el caso en España en la década de 1940, que los practicantes realizaran cirugía menor (22). En otros, las enfermeras se preparaban para lo que más tarde agruparía un conjunto de técnicas invasivas en áreas como cardiología, o bien, en salud comunitaria, donde prolongaban prescripciones preexistentes. 
En la década de 1950, los roles médicos ejecutados informalmente comienzan a formalizarse a través de 'especialidades de enfermería', las cuales reflejaban las especialidades médicas. Pero por más funcionales que fuesen al progreso tecnológico, estas especialidades no formaban parte del ordenamiento jurídico de las profesiones sanitarias.

Tanto las especialidades de enfermería como otras ramificaciones clínicas -p. ej., atención prehospitalaria, triaje, diálisis- terminarían por reunirse bajo el término baremo de EPA, lo que va a legitimar y formalizar roles hasta entonces dados por sentado. Ignorar estas prácticas no sólo invisibilizaba una carga importante del trabajo de las enfermeras, además requería un sistema informal de colocación con colegas de más experiencia y les exponía a conflictos por práctica ilícita de la medicina.

En países con carencias importantes de médicos, la enfermera hospitalaria evaluaba problemas emergentes y estimaba qué medicamentos y dosis era probable que indicara el médico; la decisión era respaldada ex post por el médico, aunque eso generaba tensiones, especialmente si el cuadro se agravaba producto de la decisión de la enfermera. Este escenario era aún más delicado en establecimientos privados. Una situación similar es la de enfermeras en inmunización que consiste no solamente en planear y conservar el stock de vacunas, sino también en aplicar criterios de elegibilidad para ser vacunado y administrar vacunas sin prescripción médica (23).

Este proceso histórico de delegación ha estado marcado, por un lado, por el debilitamiento económico del sistema -reformas de enfermería resultan de reformas de financiamiento de la salud-y por otro, por una falta de personal médico o por problemas de distribución geográfica de médicos -incluyendo un desinterés por trasladarse a zonas menos lucrativas, como las que en Francia se han denominado 'espacios médicamente deshabitados' (déserts médicaux) (24).

No obstante, cabe destacar que el mundo clínico ha influido en este proceso de delegación, lo cual explica el carácter marcadamente clínico de la EPA.

\section{Metodología}

Las políticas de salud dependen de una variedad de instituciones, procesos, actores y dispositivos. Las posibilidades de transferencia de políticas (policy transfer) se visualizan con más facilidad al analizar en detalle cómo interactúan estos elementos en el lugar de origen. La metodología de análisis de políticas (policy analysis), por ejemplo, abre posibilidades para comparar entre sistemas y realizar estudios de factibilidad antes de la transferencia, en especial cuando involucra categorías ocupacionales que no siempre son equivalentes entre países, como es el caso de la enfermería -que tiene trayectorias, planes formativos y nivel de reconocimiento muy dispares- lo cual es relevante al considerar además distintas áreas lingüísticas y geopolíticas. 
En este artículo empleamos un enfoque de análisis de políticas específico para la investigación en salud $(25,26)$. Aunque no sea un estudio comparativo per se, este enfoque fue necesario dado el énfasis en tipologías de sistemas de salud y los contextos de las políticas de bienestar social, lo cual nos permitió constituir las fuentes de manera que reflejaran las especificidades de la organización de la salud en Canadá. El trabajo de compilación de documentos se basó en una búsqueda manual de fuentes primarias originales recolectadas en Montreal durante la segunda mitad de 2019 en la Biblioteca Nacional de Quebec (BAnQ), sección Archives Nationales du Québec, además de actas de debates legislativos recopiladas en el Parlamento de Quebec (Assemblée Nationale du Québec). Estas fuentes fueron complementadas con informes publicados por asociaciones profesionales relacionados con regulación, y por organismos tales como el Institut Économique de Montréal; también se incluyó correspondencia, declaraciones públicas, documentos técnicos, entre otros, del periodo 1973-2019.

La selección comenzó con una búsqueda abierta para obtener una visión general del asunto de interés que luego se hizo más específica con base en las recurrencias, siempre teniendo en mente las instituciones, procesos, actores y dispositivos, además de la dimensión de distribución del poder entre los grupos de interés. Luego, la selección fue refinada y organizada en cuatro temas construidos de manera inductiva, los cuales estructuraron la sección de resultados. Debido a limitaciones de espacio, el análisis se enfocó en los procesos circundantes al trabajo legislativo activo.

\section{Resultados}

\section{Regulación: grupos de interés, negociaciones y jurisprudencia}

El pilar de esta política ha sido la regulación. Esto es un aspecto para considerar durante todo el trabajo legislativo como también en la interpretación, implementación y actualización de las regulaciones más específicas.

El interés en esta regulación surge porque la práctica tiende a sobrepasar la 'jurisdicción’ (27) establecida para la profesión. A menudo, la regulación ha sido motivada por cambios en las necesidades de salud, por cambios en la formación profesional, o bien para introducir una nueva tecnología. Si la regulación preexistente se interpreta de forma estricta, la práctica puede ser considerada ilícita ya que esta expansión puede involucrar otras jurisdicciones profesionales, aun si la naturaleza práctica del trabajo lo exige.

Un aspecto que tiende a olvidarse en los debates es el interés público. Si la práctica ampliada es legitimada de jure, significa que a la ciudadanía le estarán garantizados profesionales idóneos. Por esta razón, la práctica 'avanzada' corresponde a una categoría particular de enfermería no a los estudios generalistas. Es una constante que en las negociaciones y debates estén no sólo los intereses sobre la política misma, sino también los de los políticos y los de las profesiones, las que se constituyen en grupos de interés para asegurar y/o elevar su posición en el orden socioeconómico $(28,29)$. Se debe perseverar en el interés público tanto en la 
confección de la ley como en el desarrollo de las regulaciones técnicas, pues coexisten intereses contradictorios, como las dificultades para combinar experticia técnica con neutralidad política, y una disminución del altruismo que alguna vez caracterizó a las profesiones $(28,30)$. Sobre este aspecto es iluminador analizar la Ley 90 de 2002 de Quebec, conocida como Código de las Profesiones, que armonizó la legislación con el ejercicio real de unas once profesiones.

Los archivos del Parlamento muestran que ya en la década de 1970 hubo una inquietud por los procedimientos médicos delegados en las enfermeras sin la necesaria formalización, discusión que se intensificó en 1973 en la Cámara cuando apareció por primera vez en la legislación la figura de la enfermera como se conoce hoy (art. 36) (31). En esa década también se discutió la práctica delegada, en especial en zonas remotas. Si bien existieron programas formativos para quienes ejercían un rol ampliado, la EPA no logró prosperar luego de que el gobierno estimara un superávit de profesionales médicos, sumado a la tardanza en legislar debido a circunstancias sociopolíticas en Quebec en los años 70. Además, la remuneración para estas enfermeras no fue adaptada a un nivel de autoridad superior (32). Igualmente, en provincias canadienses de habla inglesa donde sí pudieron establecerse, las enfermeras de práctica avanzada tendieron a desaparecer en la década de 1980. A pesar de su afinidad cultural con Estados Unidos, donde la práctica avanzada había prosperado desde mediados de siglo, las provincias angloparlantes no lograron implantar permanentemente esta figura debido a los esquemas de financiamiento y a la falta de una legislación específica (2). Este fenómeno estuvo mediado por el despertar feminista en la década de 1970 y su eco en las expectativas de paridad salarial.

En 1997 una primera reforma, que pretendía impulsar el sistema de salud hacia el siglo XXI fue abortada por falta de consenso. Al retomarse el debate en 2002, se adoptó un principio de flexibilidad legislativa que evitaba una delimitación estricta de campos profesionales. Como señalara el entonces Ministro de Justicia, Paul Bégin (33), este proyecto previó "un nuevo uso compartido de los campos de ejercicio profesional en el área de salud" como también actividades "reservadas" para distintas profesiones "de manera de responder mejor a las necesidades de la población". Por otra parte, según consta en el acta, el proyecto de ley establecía "un marco que permitirá autorizar a profesionales además de los médicos, específicamente enfermeras y enfermeros, a ejercer ciertas actividades médicas" (33).

Aunque se percibió en general como un avance en autonomía, las actividades delegadas fueron definidas por el Colegio Médico como consta en las actas del Parlamento: "estas actividades [delegadas en enfermería] podrán ser verificadas por un comité o un médico designado por el Directorio del Colegio Médico de Quebec”. Así también se dispusieron las condiciones para realizar los actos delegados y cómo supervisarlos. En efecto, el texto del nuevo Código de las Profesiones se acompañó de una guía de interpretación de la ley ${ }^{3}$ para socializar esas condiciones ${ }^{4}$. 
Presidido por un representante del Colegio Médico de Quebec, un comité designado por el Ministerio ('Comisión Bernier') analizó las profesiones de salud durante tres años, cuyo informe condujo al proyecto de ley. Según reporta la entonces presidenta del Colegio de Enfermeras de Quebec, Gyslaine Desrosiers, durante el análisis se llevó a cabo una gira por toda la Provincia de Quebec, en que Desrosiers se reunió con unas 3.000 enfermeras para acordar los términos de negociación del proyecto (34). Una de las preocupaciones manifiestas era la participación de la auxiliar de enfermería en la terapia intravenosa, tema en que se requería adhesión mayoritaria. Asimismo, al aprobarse la ley se requirió una reglamentación específica sobre la EPA que fijara criterios para obtener la certificación correspondiente. Esto requirió definir en qué áreas clínicas se desarrollarían roles avanzados (inicialmente, neonatología, nefrología y cardiología), negociar con el Colegio Médico las tareas a delegar y con la Asociación de Auxiliares de Enfermería para convenir los actos autorizados.

La nueva ley exigía además trabajar con la Oficina de Profesiones, lo que permitiría aplicar dispositivos legislativos en el ejercicio práctico. Si bien este enfoque podría parecer bastante 'metropolitano' adecuado para grandes ciudades como Montreal, las zonas remotas representaban un caso especial dado que sus residentes rara vez recibían atención médica y que las decisiones de la enfermera no siempre contaban con apoyo de otros profesionales. No obstante, esta moción no fue fructífera.

Temiendo un bloqueo de parte del Colegio Médico, el Ministerio de Salud decidió discutir la creación de la EPA directamente a nivel de la legislación, no de normas ministeriales (35). Así, el Ministerio hizo una alianza con la Oficina de Profesiones, en tanto el Colegio Médico debía negociar con el Colegio de Enfermeras pero incluyendo la Asociación de Médicos Generalistas con quienes las enfermeras compartían más roles. Las enfermeras recurrieron también a asociaciones de médicos especialistas, principalmente aquéllas en que se proponían especializaciones de enfermería: neonatología, nefrología, cardiología y psiquiatría, recibiendo el apoyo sólo de las tres primeras (35).

Tras una era de marcada reticencia, en 2005 el Colegio Médico apoyó la creación de estas tres áreas de enfermería avanzada, estableciendo la facultad de prescribir medicamentos y tratamientos. Pese a ciertos intentos por demostrar que la enfermería psiquiátrica constituiría en sí misma una forma de práctica avanzada (36), su reconocimiento se vio trabado debido a planes por monopolizar la psicoterapia para los psiquiatras y psicólogos. Efectivamente, un consenso reciente indica que ciertas intervenciones - de la enfermería y otras profesiones- se aproximan a la psicoterapia, tales como el acompañamiento, la readaptación o la intervención en crisis, pero no constituyen un tratamiento como tal $(37,38)$, razonamiento que se empleó para no considerarlas como práctica avanzada.

Los detalles de la negociación de la Ley $\mathrm{N}^{\circ} 90$ se conocieron años más tarde (35). Salió a la luz, por ejemplo, el estatus hegemónico de la profesión médica, que en Canadá define y domina el campo ocupacional de otras profesiones a la vez que activa una movilización gremial cada vez que se anuncian reformas a la gestión del sistema de salud. Asimismo, la adopción 
relativamente tardía de la EPA en Quebec no se explica sino por la reticencia de la colectividad médica. Al evaluar la organización del sistema es evidente que la medicina constituye el núcleo de las prestaciones, en particular el diagnóstico médico que es de donde derivan muchas de las tareas clínicas y organizativas de otros profesionales, siendo las reformas un momento coyuntural para controlar sus jurisdicciones (27). Aun al delegar parcialmente la prescripción de medicamentos, el diagnóstico - por excelencia, el más intelectual de los actos médicos-sigue siendo celosamente protegido, políticamente cargado y esencialmente indelegable.

\section{La facultad de prescribir y el problema del diagnóstico}

Como se ha discutido, la EPA se ha fortalecido mediante la delegación. Es en situaciones de extrema precariedad que la enfermería se ha presentado como un recurso práctico, mediando la autorización de las élites médicas. Incluso hoy, la delegación de autoridad es tema central de muchos debates relacionados con la autonomía en la práctica avanzada.

Asimismo, la expansión de su papel ha generado temor entre las propias enfermeras a que los fundamentos de su disciplina puedan ser desplazados por esta medicalización progresiva $(10,14)$, aun si la EPA incluye muchas actividades de enfermería, no sólo las delegadas (39).

A pesar de esta delegación, y a diferencia de la situación en Ontario, el diagnóstico de enfermedades sigue siendo exclusivo de la profesión médica (40). De hecho, las directrices sobre la EPA estipulan que la enfermera puede iniciar procedimientos diagnósticos y "determinar problemas de salud comunes" (41 p.10), pero no diagnosticar enfermedades (39).

Esta decisión sugiere una negación insidiosa de la práctica de enfermería, como extensión de lo que Stein en su momento denominó "el juego del doctor y la enfermera" (42). Stein describió este juego de poder indicando, por ejemplo, que la investigación clínica de la enfermera era tolerada siempre y cuando ella fuera pasiva y sumisa a la autoridad médica. Tras varias décadas esta dinámica aún se observa en diferentes niveles y matices. Justificado bajo la premisa de 'colaboración', el que la enfermera de práctica avanzada pueda iniciar procedimientos de diagnóstico, pero no diagnosticar enfermedades, bien puede perpetuar dinámicas de dominación institucionalizadas históricamente. Si consideramos el diagnóstico como herramienta de clasificación que subraya la autoridad del médico y consolida su control biopolítico $(43,44)$, el temor a delegarlo puede fundarse en un eventual socavamiento de la profesión.

Sin embargo, en los debates públicos recientes en Quebec se ha puesto en duda que las enfermeras de práctica avanzada no deban diagnosticar enfermedades. Desde entonces se están revisando las directrices para la enfermería de atención primaria, y es probable que pronto se amplíen aún más sus facultades. 
La cuestión del diagnóstico hace parte de un problema más amplio de autonomía de las enfermeras, que históricamente han sido sometidas a la autoridad médica (42,45). Esta dinámica abarca también la invisibilidad estructural del trabajo del cuidado, partiendo por relaciones de clase y género (46-48). Si bien el discurso de 'experticia enfermera' responde a la voluntad de formalizar el trabajo del cuidado y equilibrar las relaciones interprofesionales, esto sigue dependiendo de las élites médicas y sometiendo a la enfermería a una medicalización progresiva. Vale recordar que los primeros rastros de la EPA no se encuentran en las leyes ni en las guías clínicas, sino en registros y narraciones orales sobre los primeros contingentes de enfermeras británicas en Canadá desde la década de 1890 (11).

\section{EPA en el sistema de profesiones}

La política de EPA no puede examinarse sin considerar el sistema sanitario en su conjunto, pues no es la única profesión que avanza hacia la jurisdicción médica. También lo han hecho otras profesiones establecidas como la de farmacéutico y otras en proceso de institucionalización como la de quiropráctico. Algo que las enfermeras, los farmacéuticos y los quiroprácticos tienen en común es el largo proceso sociopolítico que han debido sobrellevar para lograr cierto nivel de acuerdo con la medicina durante las reformas.

Al analizar la formación del farmacéutico, único profesional que dedica su formación entera al medicamento, encontramos que irónicamente este profesional no tiene la facultad de prescribir (49). A pesar de ello, por beneficiar al paciente debe actuar de buena fe cuando las prescripciones son ilegibles, cuando el paciente olvida la prescripción, cuando las dosis son insuficientes para llegar al próximo control médico, ante problemas comunes y cualquier situación que revista un riesgo asociado a la dosificación, interacción o falta de fármacos. Si bien en Canadá no existe una certificación de farmacéutico especialista, desde 1989 se ha autorizado tal denominación a quienes lleven a cabo un programa de residencia o se hayan especializado en el extranjero. Así también, en la Provincia de Manitoba existe un sistema de práctica 'ampliada' para farmacéuticos que traten pacientes bajo medicación compleja. Englobando estas distintas variantes, este desarrollo se ha denominado también 'práctica avanzada' o simplemente práctica especializada.

Dado que en Canadá - como país federal- coexisten distintos sistemas sanitarios, el acceso a la práctica avanzada del farmacéutico no es homogéneo. Sin embargo, el requisito general es la realización de una residencia posterior a la titulación, en la cual trabaja colaborativamente con médicos y/o enfermeras de práctica avanzada, sea en un modelo generalista o en alguna especialidad.

En cambio, la profesión de quiropráctico ha librado una batalla de décadas ante el oligopolio de la medicina y la fisioterapia ${ }^{5}$, tanto por clientes como por esquemas de financiamiento. Si bien el espectro de técnicas del quiropráctico incluye acupuntura, crioterapia, terapia de calor externo, tracción, terapia manual, masaje terapéutico, estimulación nerviosa eléctrica transcutánea, terapia de ultrasonido, entre otras (50), sigue siendo muy debatido si es que 
estas técnicas pueden ir, en ciertos casos, acompañadas de medicamentos. Pero también es necesario considerar que su campo varía mucho entre países. En general, la profesión se define como no intervencionista, ni médica ni quirúrgicamente (51), aunque esta definición comienza a revertirse mediante el uso directo y no supervisado de fármacos, sobre todo analgésicos, particularmente en Suiza y en Estados Unidos. La denominación de 'quiropráctico médico' comienza también a utilizarse esporádicamente para referirse al quiropráctico de práctica avanzada (52). Si bien es una práctica acotada, ceñida a medicamentos de venta libre, hasta ahora no parece haber un interés por medicalizar la profesión, probablemente debido a un ethos profesional históricamente contrapuesto al de la medicina científica. No obstante, esto ocurre en paralelo a la ampliación del rol del kinesiólogo en el Reino Unido, con licencia para prescribir un amplio rango de fármacos incluyendo opioides (53).

Cabe mencionar el hito importante que constituyó la autorregulación de la profesión de quiropráctico en Ontario en 2006. Esto resolvió una larga disputa sobre si la medicina es o no la institución idónea para opinar sobre teorías que nunca llega a estudiar (54), tales como la teoría en que se basa la acupuntura. Un debate similar existe en Quebec sobre la osteopatía. La Asociación Canadiense de Evaluadores Quiroprácticos sostiene que parte del problema se producía precisamente por el control médico sobre otras profesiones, modelo que conducía a una percepción exagerada de falta de médicos (54), en especial, cuando las reformas propiciaban un mejor aprovechamiento de profesionales no médicos.

El reacomodo de las profesiones en esta ecología interprofesional depende mayoritariamente de una capacidad para contrarrestar las estrategias de apropiación del diagnóstico, como también del uso de fármacos en su repertorio de servicios (28). A su favor, la profesión médica en Canadá parece ser mucho más abierta a la crítica del público que sus vecinos más cercanos, en Estados Unidos (29), a pesar de su dominancia en el proceso legislativo, en las negociaciones con otras profesiones y en su capacidad para influir en la opinión pública. Aun así, es necesario considerar los exhortos permanentes de The World Medical Association, como uno emitido en 2019 en que sostiene que ante la escasez universal de médicos, promover la formación de enfermeras y otros profesionales de la salud ${ }^{6}$ es negar a los pacientes una salud de calidad (55). Asimismo, su presidente F. Montgomery señala que "el sector salud incluye un trabajo en equipo liderado por el médico. [...] La relación médico-paciente requiere un médico, no un sustituto o un subrogante" (55), lo cual, para Montgomery, se contraviene al optar por delegar tareas en personal menos cualificado. Cabe recordar que la profesión médica es la pieza central del modelo biomédico.

Para una visión alternativa al medicocentrismo, la OMS ha abogado por un modelo interprofesional que permita emplear las nuevas competencias de los profesionales producto de los cambios en la formación en universidades, que aunque a la larga tiende a favorecer la medicalización, en una dimensión sociopolítica viene a reivindicar una legitimidad de facto. 


\section{Lo reciente en Canadá: una clínica de enfermeras avanzadas}

Además de los servicios convencionales, cambios recientes en la EPA en Canadá han estimulado una serie de iniciativas para dar más acceso a la salud. En 2007 la Provincia de Ontario anunció algo inusual: la creación de 25 clínicas dirigidas por profesionales de enfermería (56). La organización de estas clínicas descansa en la experticia de enfermeras de práctica avanzada. Aunque ellas desempeñan un papel central -coordinan, administran y ejecutan los actos inherentes a su ejercicio- estas clínicas emplean un enfoque interprofesional que además requiere una alianza con el usuario. Además de enfermeras de práctica avanzada, las clínicas incluyen también enfermeras sin especialización, auxiliares de enfermería, médicos, nutricionistas, trabajadores sociales, entre otros profesionales (56).

Estas clínicas son organizaciones sin ánimo de lucro financiadas con fondos públicos. Cada una tiene una junta directiva compuesta por enfermeras de práctica avanzada, profesionales de servicios sociales y de salud, además de ciudadanos de la comunidad (57). Es interesante puntualizar que la entidad que congrega a las enfermeras de práctica avanzada (Nurse Practitioners' Association of Ontario, NPAO) sugiere que al menos el 51\% del directorio de estas clínicas sea de EPA (57). En otras palabras, la posición estratégica de estos profesionales en Ontario se extiende más allá del cuidado del paciente, formando parte de la gobernanza en atención primaria.

Por su parte, en Quebec, el modelo que sostiene a la práctica avanzada en atención primaria es diferente: se basa en los Centros Locales de Servicios Comunitarios (CLSC) y, más recientemente, en el modelo de Grupos de Medicina Familiar (GMF) que son organizaciones privadas financiadas por el sistema público (58); a su vez conforman la instancia que capta la mayor parte de enfermeras de práctica avanzada en atención primaria en Quebec. El gobierno provincial (59) define los GMF como "grupos de médicos de familia que trabajan en estrecha colaboración con otros profesionales de la salud". No obstante, Quebec no cuenta oficialmente con ninguna clínica administrada por enfermeras de práctica avanzada financiada en su totalidad por el sistema público; y vale mencionar que la financiación depende de la administración provincial y no del gobierno federal (58).

Se ha sugerido además que los GMF contribuyen a mantener una organización medicocéntrica, lo que relega a las enfermeras de práctica avanzada a un rol de subalternas del médico (60) en lugar de aprovechar su campo de ejercicio. Los cambios recientes que ha visto la EPA en Quebec tienden a otorgarle una libertad mucho mayor, pero la realidad práctica siempre se pacta mediante un acuerdo formal con los médicos con quienes se trabaja $(61,62)$.

A pesar de ello, la política permite otras iniciativas de enfermeras de práctica avanzada en atención primaria, como la Clínica SABSA, que sigue un modelo cooperativo de economía solidaria. Aunque aún muy minoritarios, los modelos cooperativos son cada vez más reconocidos, y a nivel mundial han sido documentadas varias iniciativas (63). 
La Clínica SABSA comenzó sus actividades en 2011, aunque se inauguró oficialmente en 2014. Inicialmente tenía la intención de proporcionar atención médica y servicios a la población denominada 'vulnerable' y marginada de la ciudad de Quebec, como también a personas que viven con VIH o Hepatitis C (64). Al igual que las clínicas en Ontario, la Clínica SABSA funciona bajo la administración de enfermeras avanzadas para proporcionar tanto atención de enfermería como servicios interprofesionales. Estos servicios se brindan en la clínica, o bien, externamente a través de distintas asociaciones. El informe más reciente indica que menos del $5 \%$ de las consultas realizadas por enfermeras conduce a una referencia a otro profesional de salud (65), lo cual reflejaría su nivel de resolutividad.

Una diferencia importante con las clínicas de enfermeras en Ontario es que la financiación de la Clínica SABSA no proviene totalmente de fondos públicos. Entre 2011 y 2014, la clínica sobrevivió gracias a la contribución de ciudadanos individuales y la participación de voluntarios (65). La ampliación de los servicios también ha sido posible gracias al apoyo del sector privado, tales como sindicatos y bancos (65). Después de varios años de negociación por el reconocimiento de sus actividades, la Clínica SABSA obtuvo en 2016 un primer financiamiento público para establecer el puesto de enfermera de práctica avanzada en atención primaria (65). Hasta la fecha, la clínica se beneficia de fondos temporales proporcionados por el sector público, a la vez que lleva a cabo actividades de autofinanciamiento para garantizar su existencia.

Los casos de Quebec y Ontario, siendo provincias colindantes, permiten ilustrar la complejidad administrativa y los problemas políticos en que se sitúa y desarrolla la EPA. Aunque las iniciativas presentadas en este artículo figuran positivamente en el imaginario social, aún existe una dinámica de competición entre las enfermeras avanzadas en atención primaria y la profesión médica (66). Una razón es la superposición histórica del campo ocupacional (45), lo cual pudiese amenazar el diálogo interprofesional y la complementariedad de las prácticas $(45,66)$. En tanto la evidencia sugiere que los servicios de atención primaria pueden ser prestados de manera segura y efectiva por enfermeras $(67,68)$, el potencial de las clínicas dirigidas por enfermeras de práctica avanzada abre nuevas perspectivas de investigación y permite repensar la organización de la salud (65).

Sin adoptar una lógica de competencia, la aparición de enfermeras de práctica avanzada en atención primaria como actores clave facilitaría la reestructuración de la atención y los servicios de salud primaria. Esta reestructuración podría tener como efecto la redefinición de los límites interprofesionales, un distanciamiento la atención del medicocentrismo que la caracteriza y una mayor integración del llamado enfoque 'biopsicosocial' (65).

\section{Discusión y conclusiones}

En este artículo analizamos la institucionalización de la EPA como política de salud. Dos factores gravitan en sus avances y retrocesos. Por una parte, la distribución geográfica del personal médico que tiende a concentrarse en los centros urbanos, junto con las necesidades sin 
cubrir de segmentos de la población. Por otra parte, la percepción de inseguridad económica que resurge periódicamente vuelve la atención hacia las tareas y áreas delegadas en la enfermería a fin de recuperarlas. Otros factores como el escepticismo disciplinar de las propias enfermeras y los esquemas de financiación también han afectado este proceso.

Es difícil evaluar el impacto completo de esta política, en tanto atribuir resultados -favorables o desfavorables-a las intervenciones de las enfermeras podría ser erróneo. Si bien su resolutividad es alta considerando la tasa de referencia a otros profesionales, pudiese haber también una reticencia a referir pacientes al ser un aspecto clave en la evaluación de la política. Las críticas apuntan a los costos asociados a la eventual duplicidad de atenciones cuando se llega a producir una referencia. No obstante, estos casos son minoritarios.

En Canadá, la mayor autonomía de la enfermería significa más cuidados para los pacientes, que con la práctica avanzada son a la vez cuidados de enfermería y cuidados médicos. Asimismo, la jurisprudencia creada para un actuar interprofesional ha venido a proteger tanto a las enfermeras como a los usuarios. Estas modificaciones jurídicas se comprenden mejor al enfocarse en las transformaciones del sistema de profesiones de manera global, reorganización aún no terminada. $\mathrm{Si}$ esto reconoce los roles y tareas que las profesiones han desarrollado de manera informal por proteger al paciente, lo mismo cuenta para las ocupaciones técnicas que hoy tienen menos supervisión cercana, pues ambos grupos han cubierto históricamente vacíos en los servicios de salud.

Aun cuando la profesión médica en Canadá es, en general, mucho más abierta a la crítica que en otros lugares deben considerarse las dificultades de negociar con este grupo dominante en la formulación de las políticas. Pese al silencio en el conflicto, aún queda por resolverse cierta disonancia en los círculos académicos ya que el avance de la EPA radica precisamente en su asimilación a la medicina. Y vale mencionar que la noción de enfermería 'avanzada' resulta peyorativa para las enfermeras que deciden no seguir esta formación.

Un aspecto relevante es la infraestructura de la política de salud conformada por diferentes dispositivos de menor rango que derivan de la política, tales como guías clínicas (en particular desde 2014) que reemplazaron la supervisión directa en los inicios, becas de estudio para atraer enfermeras a esta área, incentivos económicos tanto a enfermeras como a médicos a fin de apoyar la implantación, el visado de parte de la Oficina de Profesiones de Quebec, la adaptación de regulaciones deontológicas de las colectividades de enfermeras y legales para incluir la práctica avanzada, en especial respecto a la autoridad para prescribir $(41,61)$ así como también la coordinación con los Ministerios de Salud y de Educación a fin de ajustar los planes de estudio y procurar la creación de puestos de trabajo. Debido a esta complejidad aún no se han resuelto completamente las dificultades de implantación de enfermeras de práctica avanzada en los servicios, pues la certificación por sí sola no neutraliza la resistencia interna en los equipos $(2,68,69)$. 
Otro problema subyacente es el de la remuneración ya que al aumentar en extensión los estudios se asemejan a los del médico general. Así también, aunque el surgimiento de la EPA surge como respuesta a la precariedad de los sistemas de salud, los roles avanzados contribuirían a la creciente medicalización del sistema y de paso a mantener el estatus quo más que propiciar un cambio 'paradigmático' en salud.

Esta reflexión de los factores clave para la formulación de la política sanitaria en Estados Unidos y Canadá puede ser retomada por futuras investigaciones para proyectar una nueva agenda sobre las profesiones de salud o para estudiar la eventual transferencia a otros sistemas de salud de esta política de atención con EPA.

\section{Consideraciones éticas}

Los autores declaran que este artículo es inédito y se hacen responsables por su contenido.

\section{Contribución de los autores}

Artículo de investigación producido durante una estadía de investigación del primer autor en la Universidad de Quebec (Canadá) en agosto de 2019, en colaboración con el segundo autor.

\section{Financiación}

Recibió financiamiento de la Research Foundation Flanders de Bélgica.

\section{Referencias}

1. Betancourt MT, Roberts KC, Bennett TL, Driscoll ER, Jayaraman G, Pelletier L. Monitoring chronic diseases in Canada: The chronic disease indicator framework. Chronic Dis Inj Can. 2014;34(sup1):1-30. https://pubmed.ncbi.nlm.nih.gov/24898593/

2. Rioux-Dubois A. L'intégration et la négociation du rôle de l'infirmière praticienne en soins de santé primaires en contexte de collaboration interprofessionnelle. Tesis doctoral. Ottawa: Université d'Ottawa; 2019.

3. Sevilla S. The implementation of Advanced Practice Nursing in Catalonia. Tesis doctoral. Barcelona: Universidad de Barcelona; 2018.

4. Bochel H, Bochel C. Making and implementing public policy. Key concepts and issues. London: Palgrave; 2018.

5. Canadian Nurses Association. The Canadian Nurse Practitioner Initiative: A 10-Year Retrospective; 2016. https://www.cna-aiic.ca/-/media/cna/page-content/pdf-en/canadian-nurse-practitioner-initiativ e-a-10-year-retrospective.pdf 
6. American Association of Nurse Practitioners. Nurse practitioners, fact sheet; 2019. https://www.aanp.o $\mathrm{rg} /$ about/all-about-nps/np-fact-sheet

7. Canadian Nurses Association. Nurse practitioners - Untapped resources; 2018. https://cna-aiic.ca/-/med $\mathrm{ia} / \mathrm{cna} /$ page-content/pdf-en/2019-nurse-practitioners-untapped-resource.pdf

8. American Association of Nurse Practitioners. What's a Nurse Practitioner (NP)? 2019. https://www.aan p.org/about/all-about-nps/whats-a-nurse-practitioner

9. College of Nurses of Ontario. Practice standard: Nurse Practitioner; 2019. http://www.cno.org/globalas sets/docs/prac/41038_strdrnec.pdf

10. MacDonald JA, Herbert R, Thibeault C. Advanced practice nursing: Unification through a common identity. J Prof Nurs. 2006;22(3):172-179. https://doi.org/10.1016/j.profnurs.2006.03.009

11. Kaasalainen S, Martin-Misener R, Kilpatrick K, Harbman B, Bryant-Lukosius D, Donald F, Carter N, DiCenso A. A historical overview of the development of advanced practice nursing roles in Canada. Nurs Leadersh. 2010 Dec;23:35-60. https://doi.org/10.12927/cjnl.2010.22268

12. Sheer B, Wong FK. The development of advanced nursing practice globally. J. Nurs. Scholarsh. 2008;40(3):204-211. https://doi.org/10.1111/j.1547-5069.2008.00242.x

13. Association des Infirmières et Infirmiers du Canada. L'Infirmière Praticienne. Énoncé de Position; 2016.

14. Delvin M-E, Braithwaite S, Camargo P. Canadian nurse practitioner's quest for identity: A philosophical perspective. Int J Nurs Sci. 2018;Apr(5):110-114. https://doi.org/10.1016/j.ijnss.2018.03.001

15. Ayala R. El concepto de situs ocupacional: Por qué esta propuesta no es plausible: Una explicación sociológica. Cienc Enferm. 2015;21(3):133-141. http://dx.doi.org/10.4067/S0717-95532015000300 012

16. Ayala R, Binfa L, Vanderstraeten R, Bracke P. Exploring historical conflicts between midwives and nurses: A perspective from Chile. J Interprof Care. 2015 May;29(3):216-222. https://doi.org/10.31 $09 / 13561820.2014 .956863$

17. Anastakis D. Industrialization in Canada. In the Canadian Encyclopedia; $2019 \mathrm{https}: / / \mathrm{www}$. thecanadi anencyclopedia.ca/en/article/industrialization

18. Canadian Nurses Association. Exploring new roles for advanced nursing practice. A Discussion Paper; 2005.https://www.cna-aiic.ca/ /media/cna/page-content/pdf-en/exploring_new_roles_anp-05_e.pdf

19. Keeling AW, Bigbee JL. The history of advanced nursing practice in the United States. In: Hamric A, Spross J, Hanson C (eds). Advanced practice nursing: An integrative approach, $3^{\text {rd }}$ ed. St. Louis: Elsevier Saunders; 2005.

20. Keeling AW. A brief history of advanced practice nursing in the United States. In: Hamric A, Spross J, Hanson C (eds). Advanced practice nursing: An integrative approach, $3^{\text {rd }}$ ed. St. Louis: Elsevier Saunders; 2005.

21. Durán MA. Medicalización, Higienismo y Desarrollo Social en Chile y Argentina, 1860-1918. Tesis de maestría. Santiago: Universidad de Santiago; 2012. 
22. Boletín Oficial del Estado [España] del 5 de diciembre 1945, Boletín 339 artículo 7. https://www.boe .es/datos/pdfs/BOE//1945/339/A03334-03343.pdf

23. Stewart AM, Lindley MC, Cox MA. State law and standing orders for immunization services. Am. J. Prev. Med. 2016 May;50(5):e133-e142. https://doi.org/10.1016/j.amepre.2015.10.003

24. Fournier C. Les pratiques pluriprofessionnelles dans des maisons de santé libérales : attentes, contenus, interactions et instruments mobilisés. In: Fillaut DF, Hontebeyrie T (eds). Intervenir en première ligne. Les professions de santé libérales face au défi de la proximité. Paris: L’Harmattan; 2016. p.109-122.

25. Burau V. Comparative Health Research. In : Saks M, Allsop J (eds). Researching Health. Qualitative, quantitative and mixed methods. London: Sage; 2019, p.453-471.

26. Burau V, Blank RH. Comparing health policy: An assessment of typologies of health systems. Journal of Comparative Policy Analysis. 2006;8(01):63-76. https://doi.org/10.1080/13876980500513558

27. Abbott A. The system of professions: An essay on the division of expert labor. Chicago: University of Chicago Press; 2014.

28. Saks M. Professions and the public interest: Medical power, altruism and alternative medicine. London: Routledge; 2005.

29. Ahmed H, Brown A, Saks M. Patterns of Medical Oversight and Regulation in Canada. In: Chamberlain JM, Dent M (eds). Professional Health Regulation in the Public Interest: International Perspectives. Bristol: Policy Press; 2018. p.135-161.

30. Ayala R. Towards a sociology of nursing. Singapore: Palgrave Macmillan; 2020.

31. Code des professions. Loi des infirmières et infirmiers, LQ 1973, chap. 48.

32. Ferré-Deslongchamps KL. L'infirmière praticienne spécialisée et la responsabilité civile. Tesis doctoral. Sherbrooke: Université de Sherbrooke; 2012.

33. Bégin P. Projet de Loi $\mathrm{N}^{\circ}$ 90. Loi modifiant le Code des professions et d'autres dispositions législatives dans le domaine de la santé. Éditeur officiel du Québec; 2002.

34. Desrosiers GP. Rapport de la Présidente. Rapport Annuel 2002-2003, Ordre des infirmières et infirmiers du Québec. p 6-9.

35. Hudon R, Martin É, Perreault M. Le pouvoir médical et le défi de la collaboration interprofessionnelle. Trois cas de figure. Rech. Sociogr. 2009;50(2):321-344. https://doi.org/10.7202/038042ar

36. Goudreau J, Poirier MJ, de Montigny F. Approche clinique en soins infirmiers psychiatriques: Étude de cas d'une pratique avancée. Rech Soins Infirm. 2006;1(84):118-125. https://doi.org/10.3917/rsi. 084.0118

37. Association des Médecins Psychiatres du Québec. En quoi consiste le rôle du médecin psychiatre?; 2019. https://ampq.org/faq/

38. Ordre des Psychologues du Québec. Qu'est-ce que la psychothérapie?; 2019. https://www.ordrepsy.q c.ca/qu-est-ce-qu-un-psychotherapeute- 
39. Publications du Québec. Règlement sur les infirmières praticiennes spécialisées; 2019. http://legisque bec.gouv.qc.ca/fr/ShowDoc/cr/M-9,\%20r.\%2023.1

40. College of Nurses of Ontario. Practice standard: Nurse Practitioner; 2019. http://www.cno.org/globala ssets/docs/prac/41038_strdrnec.pdf

41. Ordre des infirmières et infirmiers du Québec y Collège des Médecins du Québec; Pratique clinique de l'infirmière praticienne spécialisée en soins de première ligne. Lignes directrices; 2019. https://umfs udlanaudiere.files.wordpress.com/2014/06/lignes-ips-prem-ligne.pdf

42. Stein LI. The doctor-nurse game. Arch. Gen. Psychiatry. 1967;16(6):699-703. https://doi.org/10.1001 /archpsyc.1967.01730240055009

43. Jutel A. Sociology of diagnosis: A preliminary review. Soc Health Ill. 2009;31(2):278-299. https://do i.org/10.1111/j.1467-9566.2008.01152.x

44. Conrad P. The Medicalization of Society. Baltimore: John Hopkins; 2007.

45. Benoit M, Pilon R, Lavoie A, Pariseau-Legault P. Chevauchement, interdépendance ou complémentarité ? La collaboration interprofessionnelle entre l'infirmière praticienne et d'autres professionnels de santé en Ontario. San Pub. 2017;5(29):693-706. https://doi.org/10.3917/spub.175 .0693

46. Saillant F. La part des femmes dans les soins de santé. Revue Internationale d'Action Communautaire. 1992;28(68):95-106. https://doi.org/10.7202/1033807ar

47. Chadoin M, Messing K, Daly T, Armstrong P. “Si ce n'est pas documenté, ça n'a pas été fait”: quand les indicateurs de gestion escamotent le travail invisible des femmes. Pistes. 2016:18(2). https://do i.org/10.4000/pistes. 4830

48. Ayala RA, Fealy GM, Vanderstraeten R, Bracke P. Academisation of nursing: An ethnography of social transformations in Chile. Int J Nurs Stud. 2014;51(1):603-611. https://doi.org/10.1016/j.ijnurstu.20 13.08.010

49. Marceau N, Florice M. Spécialisation et pratique avancée de la pharmacie dans le monde. Pharmactuel. 2019;52(1). https://pharmactuel.com/pharmactuel/index.php/pharmactuel/article/view/1268/1106

50. Canadian Society of Chiropractic Evaluators. Treatment modalities; 2019. https://www.thecsce.ca/en/ articles/treatment-modalities

51. Emary PC, Stuber KJ. Chiropractors' attitudes toward drug prescription rights: A narrative review. Chiropr Man Therap. 2014(Sep);22:34. https://doi.org/10.1186/s12998-014-0034-7

52. Smith M, Bero L, Carber L. Could chiropractors screen for adverse drug events in the community? Survey of US chiropractors. Chiropr Osteopat. 2010;18(30):1-8. https://doi.org/10.1186/1746-1340 $-18-30$

53. Chartered Society of Physiotherapy. Medicines, prescribing and injection therapy. Medicines use, prescribing and injection therapy are within the scope of the UK physiotherapy profession; 2019. https://www.csp.org.uk/professional-clinical/professional-guidance/medicines-prescribing-an d-injections\#prescribing 
54. Canadian Society of Chiropractic Evaluators. The role for chiropractic experts in independent assessment; 2019. https://www.thecsce.ca/en/articles/the-role-for-chiropractic-experts-in-independe nt-assessment

55. World Medical Association. WMA leader's warning over substituting physicians; 2019 May 15. https: //www.wma.net/news-post/wma-leaders-warning-over-substituting-physicians/

56. Legislative Assembly of Ontario. Introduction to Nurse Practitioner-Led Clinics; 2010. http://www.on tla.on.ca/library/repository/mon/24005/301596.pdf

57. Nurse Practitioners' Association of Ontario. Nurse Practitionner-Led Clinics; 2019. https://npao.org/a bout-npao/clinics/

58. Breton M, Jevesque JF, Pineault R, Hogg W. L'implantation du modèle des groupes de médecine de famille au Québec: potentiel et limites pour l'accroissement de la performance des soins de santé primaires. Pratiques et organisation des soins. 2011;2(42):101-109. https://doi.org/10.3917/pos.422 .0101

59. Gouvernement du Québec. Groupe de médecine de famille (GMF), groupe de médecine de famille universitaire (GMF-U) et super-clinique; 2019. https://www.quebec.ca/sante/systeme-et-services-de -sante/organisation-des-services/gmf-gmf-u-et-super-clinique/

60. D’Amour D. Collaboration entre les infirmières et les médecins de famille. Pourquoi et dans quelles conditions? Ruptures. 2001;8(1):136-145.

61. Ordre des infirmières et infirmiers du Québec y Collège des Médecins du Québec; Pratique clinique de l’infirmière praticienne spécialisée en soins de première ligne. Lignes directrices; 2019.

62. Ordre des infirmières et infirmiers du Québec. Modèle d'entente de partenariat entre l'infirmière praticienne spécialisée et le ou les médecins partenaires. Documento de trabajo; 2019.

63. Lethielleux L, Thénot M, Deroy X. Les coopératives de santé : un nouveau sentier dans l'organisation des soins ? Management \& Avenir. 2018;2(100):189-207. https://doi.org/10.3917/mav.100.0189

64. Clinique SABSA. Historique; 2019. https://www.sabsa.ca/historique/

65. Contandriopoulos D, Brousselle A, Breton M, Sangster-Gormley E, Kilpatrick K, et al. Nurse practitioners, canaries in the mine of primary care reform. Health Policy. 2016;120(6):682-689. htt ps://doi.org/10.1016/j.healthpol.2016.03.015

66. Hepburn B. Fear-mongering about nurse practitioners. The Hamilton Spectator. 2019 Aug 8. https://www.niagarafallsreview.ca/opinion-story/9540555-bob-hepburn-fear-mongering-about-nu rse-practitioners/

67. Randall S, Crawford T, Currie J, River J, Betihavas V. Impact of community based nurse-led clinics on patient outcomes, patient satisfaction, patient access and cost effectiveness: A systematic review. Int J Nurs Stud. 2017;Aug(73):24-33. https://doi.org/10.1016/j.ijnurstu.2017.05.008

68. Laurant M, van der Biezen M, Wijers N, Watananirun K, Kontopantelis E, et al. Nurses as substitutes for doctors in primary care. Cochrane Database Syst Rev. 2018;16(7):CD001271. https://doi.org/10 .1002/14651858.CD001271.pub3 
69. Rioux-Dubois A. Le manque de clarté du rôle des infirmières praticiennes: entité sociopolitique de l'intégration et de la négociation des rôles en pratique avancée. Actas del Forum La pratique infirmière à la croisée des chemins; 2019 Agosto 30; Saint-Jérôme, Canada.

\section{Notas}

1 En general, esta formación se obtiene a nivel de Master o de una formación de Post-Master.

2 Las especialidades de enfermería, en general, están integradas en el nivel de Master.

3 Cahier Explicatif. Loi modifiant le code des professions et d'autres dispositions législatives dans le domaine de la santé de la Oficina de Profesiones de Quebec, 2003. También Application de la loi modifiant le code des professions et d'autres dispositions législatives dans le domaine de la santé, preparado para las enfermeras en 2002.

4 Si bien se está discutiendo una nueva reforma que otorgue a las enfermeras la autoridad para diagnosticar (Proyecto de Ley $\mathrm{N}^{\circ} 43$ ), es probable que se deleguen los diagnósticos más rutinarios.

5 En ciertos países fisioterapia corresponde a la profesión de kinesiólogo. En otros, es el quiropráctico quien se acerca más, en la práctica, al kinesiólogo.

6 El gobierno de Canadá cuenta con un generoso esquema de financiación de 640 plazas anuales para la formación en EPA. Ver: h ttp://publications.msss.gouv.qc.ca/msss/fichiers/2013/13-945-01W.pdf 